\title{
Why every anesthesia trainee should receive global health equity education
}

\section{De la nécessité que chaque résident en anesthésie reçoive une formation en équité en santé mondiale}

\author{
Elliot Wollner, MBBS, MPH (iD · Tyler Law, MD, MS • Kristina Sullivan, MD • Michael S. Lipnick, MD
}

Received: 7 January 2020/Revised: 21 March 2020/Accepted: 23 March 2020/Published online: 20 May 2020

(C) Canadian Anesthesiologists' Society 2020

"Education either functions as an instrument which is used to facilitate integration of the younger generation into the logic of the present system and bring about conformity or it becomes the practice of freedom, the means by which men and women deal critically and creatively with reality and discover how to participate in the transformation of their world."

- Paulo Freire - Pedagogy of the Oppressed Inequitable access to safe surgery, anesthesia, and pain services is one of the greatest contemporary global health (GH) crises, yet most North American anesthesia training programs do not adequately prepare future anesthesiologists to recognize or address this growing challenge. Five of the world's seven billion people lack access to timely and affordable surgical and anesthesia services. ${ }^{1}$ For those who can access these services, over 30 million people per year face catastrophic healthcare expenditure (i.e., when out-of-pocket healthcare costs lead to financial hardship for individuals or households). For some, the risk of dying from anesthesia-related complications is as high as one in $100 .^{2}$ Access to analgesia is similarly restricted worldwide.

Approximately $80 \%$ of the global population has either insufficient or no access to treatment for moderate or severe pain, with nearly 150 countries lacking reliable

E. Wollner, MBBS, MPH $(\bowtie) \cdot T$. Law, MD, MS .

M. S. Lipnick, MD

Division of Global Health Equity, Department of Anesthesia and Perioperative Care, University of California San Francisco, 1001 Potrero Ave, Bldg 5, Rm 3C38, San Francisco, CA 94110, USA e-mail: elliot.wollner@ucsf.edu

K. Sullivan, MD

UCSF Anesthesia Residency Program and Associate Chair for Anesthesia Education, San Francisco, CA, USA access to basic analgesics such as morphine. ${ }^{3}$ As highlighted by former World Bank President Jim Kim, inequities in access to surgical and anesthesia care represent a grave injustice, and jeopardize peoples' human right to healthcare and their ability to lead "healthier, more productive lives". ${ }^{4}$

Vast inequities in healthcare are not confined to low and middle-income countries (LMICs). In many high-income countries (HICs), vulnerable and marginalized populations face multiple barriers to healthcare, resulting in alarming health disparities. ${ }^{5,6}$ Impoverished and rural populations frequently have inadequate access to surgical and anesthesia services. ${ }^{7,8}$ Indigenous and non-English speaking populations are less likely to receive standard of care treatment. ${ }^{9}$ In some major US cities, life expectancy varies by more than 20 years between adjacent low- and high-income neighbourhoods. ${ }^{10}$ Encouraging future anesthesiologists to confront and address these issues locally and globally should be a priority for all training programs. $^{11}$

Despite the rising popularity of $\mathrm{GH}$ among anesthesiology resident trainees and urgent calls to increase access to anesthesia services for the majority of the world's population, few North American anesthesia training programs offer structured $\mathrm{GH}$ education (e.g., formalized pathways and fellowships) to prepare trainees to recognize and address these complex issues and encourage careers in $\mathrm{GH}{ }^{12}-{ }^{15}$ Interested residents are met with a disparate and oftentimes confusing career landscape; as a result, their enthusiasm for $\mathrm{GH}$ often remains underdeveloped and underutilized. Many training programs offer electives to work clinically or conduct short-term scholarly projects in LMICs, frequently and disappointingly unaccompanied by adequate formal $\mathrm{GH}$ education. ${ }^{13}-{ }^{15}$ Conversely, other specialties such as 
internal medicine and pediatrics have well established resident GH programs. ${ }^{16,17}$ Nearly half of all Accreditation Council for Graduate Medical Education (ACGME) pediatrics programs incorporate $\mathrm{GH}$ into their training curricula. ${ }^{18}$ Recently, surgical colleges have prioritized establishing GH education curricula and encouraging careers in $\mathrm{GH}^{19}$

We call for the urgent incorporation of basic GH education into all North American anesthesia training programs, the expansion of structured GH opportunities for residents and fellows seeking a career path focused on health disparities locally or globally, and the establishment of anesthesia-specific GH competencies and curricula. These can serve as steps toward improving access to safe anesthesia, analgesia, and surgical services for vulnerable and marginalized populations in all countries. Only through a more rigorous and structured approach to GH during training can we hope to develop globally conscious anesthesiologists that are equipped to effectively advocate for underserved populations and play a more central role in shaping the creation of future healthcare systems.

\section{What is global health and global health equity?}

Global health is a multidisciplinary field of research, study, advocacy, policy, and practice dedicated to improving overall health and achieving equity in health worldwide, with a focus on underserved populations. ${ }^{20}$ Despite its positive intentions, the field of $\mathrm{GH}$ and the term itself are not without problems and misinterpretation. Formerly known as "tropical medicine," GH has colonial roots that have led to contemporary challenges, including power imbalances that have resulted in the prioritization of HIC agendas and skewed benefit and recognition toward HIC collaborators.

Global health is also commonly misunderstood as international health, with implications that one must work abroad or focus on challenges exclusive to LMICs. ${ }^{21}$ Global health transcends national boundaries and attempts to reduce or eliminate health disparities wherever they happen, unconstrained by geographical location. ${ }^{11,21}$

Traditionally, GH has prioritized fields such as maternal and child health, food insecurity, poverty, infectious diseases, and environmental sustainability. Until recently, surgery, anesthesia, and pain medicine have not been included on the GH agenda, and have therefore lagged behind medicine and other disciplines in terms of $\mathrm{GH}$ research, advocacy, and education.

Recently, the definition of GH has been reframed to emphasize the concept of equity, and has subsequently been rebranded by some as "Global Health Equity"
(GHE). ${ }^{11}$ Equity is the absence of avoidable or remediable differences among groups of people, whether those groups are defined socially, economically, demographically, or geographically. Health inequities are inherently unjust and correctable. ${ }^{22}$ Global health equity intersects with the practice of social medicine, a field growing in prominence with its groundings in health disparities and the social determinants of health (Table 1). We support using the term GHE to more accurately describe the field, and use GHE for the remainder of the article.

In surgery and anesthesia, GHE initiatives have most commonly been manifested as "mission trips". This has created the misperception that the primary role for anesthesiologists in GHE exists through clinical service in an international setting. While mission trips help many patients, critics argue that some mission trips lack transparency, accountability, and cultural appropriateness, ${ }^{23}$ and also question their ethics and longterm benefit to the local population. ${ }^{24}$ With the expanded definition of GHE in mind, and greater recognition of the scope and scale of inequities relevant to perioperative care (Table 2), many have called for new and more sustainable approaches for improving global access to safe and affordable anesthesia.

\section{Why do all residents need GHE training?}

Exposure influences career paths

Approximately half of all anesthesia trainees pursue subspecialty fellowships in disciplines such as cardiac, pediatrics, obstetrics, and chronic pain. ${ }^{25}$ It is unlikely they would do so without exposure and instruction in these fields during their training. Currently, in the US and Canada, most anesthesia residents do not receive GHE education during their training, and unsurprisingly relatively few pursue careers focusing on underserved populations or health disparities, either domestically or abroad. A significant proportion of residents complete their training unaware of the magnitude of local and $\mathrm{GH}$ inequities, with limited understanding of the principles of GHE and the social determinants of health. Exposure to core GHE education and vocational experiences during training significantly increases the likelihood that trainees will go on to dedicate part or all of their careers to addressing health disparities in either HICs or LMICs. ${ }^{26}$

Relevance in all practice settings

Inequities in healthcare access, affordability, and safety exist everywhere and are relevant regardless of practice 
Table 1 Definitions of global health, social medicine, and social determinants of health

\begin{tabular}{ll}
\hline Discipline & Definition \\
\hline Global health $^{20}$ & $\begin{array}{c}\text { Global health is "an area for study, research, and practice that places a priority on improving health and achieving } \\
\text { equity in health for all people worldwide. Global health emphasizes transnational health issues, determinants, and } \\
\text { solutions; involves many disciplines within and beyond the health sciences and promotes interdisciplinary } \\
\text { collaboration; and is a synthesis of population-based prevention with individual-level clinical care." }\end{array}$ \\
Social medicine & $\begin{array}{l}\text { Social medicine seeks to understand the ways in which social factors influence health, disease, and medical practice. } \\
\text { As a field of practice, it applies this understanding to inform health interventions at the individual, community, and } \\
\text { society levels. Social medicine provides conceptual frameworks for approaching problems that otherwise seem } \\
\text { intractable or outside the domain of clinical practice. }\end{array}$ \\
$\begin{array}{c}\text { She social determinants of health are the conditions in which people are born, grow, live, work, and age. These } \\
\text { health } \\
\text { circumstances are shaped by the distribution of money, power, and resources at global, national, and local levels. } \\
\text { The social determinants of health are mostly responsible for health inequities-the unfair and avoidable differences } \\
\text { in health status seen within and between countries. }\end{array}$ \\
\hline
\end{tabular}

Table 2 Ten significant global health inequities relevant to anesthesia and pain

1 Five billion people worldwide do not have access to safe and affordable surgical and anesthesia care

2 More people die from surgically treatable conditions and unsafe surgical care than from HIV, TB, and malaria combined, yet less than 1\% of development funding for health supports delivery of anesthesia and surgery

3 Only $6 \%$ of all operations are done in LMICs, yet half of all postoperative deaths occur in LMICs

4 The anesthesia mortality rate in HIC is approximately 1:100,000, while in some LMICs it is as high as 1:100

5 Over 30 million people face catastrophic healthcare spending per year, mainly in LMICs

6150 countries are without reliable access to essential analgesics such as morphine

7 There is a large discrepancy between the global chronic pain burden and the resources allocated to address it in HICs and especially in LMICs

8 Marginalized populations are less likely to receive 'standard' anesthesia care, such as labour epidurals or analgesia prescribing, in both HICs and LMICs

9 HIC-led policies on global anesthesia and analgesia drug availability disproportionately limit access to essential medications in LMICs

10 Rural and poor populations in HICs and LMICs are less likely to have access to specialist anesthesia and pain providers

HICs = high-income countries; HIV = human immunodeficiency virus; LMIC = low- and middle-income countries; TB = tuberculosis

context or future career plans (Table 2). To offer the best possible care to our patients, we must understand the powerful role that social, environmental, economic, political, and cultural forces play in determining patients' health. Anesthesia providers, as with all healthcare professionals, must have an awareness of the systemic factors that create and sustain health inequities to best advocate for patients and provide optimal care for all patient populations.

\section{Requirement to meet current training standards}

In the US, it is an ACGME requirement that "all trainees show responsibility to patients, families and society," and are expected to identify "issues of importance to diverse patient populations and how limited resources may impact patient care and resource allocation". ${ }^{27}$ Similarly, Canada's Royal College of Physicians and Surgeons' includes "health advocate" as a core competency for anesthesiologists, requiring that physicians are able to "identify determinants of health", "respond to the needs of the communities or populations they serve by advocating with them for system-level change in a socially accountable manner", "promote policies which measure and support access to surgical care", and work to "support the mobilization of resources to effect change". ${ }^{28} \mathrm{We}$ argue that both the ACGME and the Royal College's requirements can only be achieved through an understanding of basic GHE principles through formalized education. ${ }^{29}$ Rotating through safety net hospitals or completing short electives in resourceconstrained settings does not equate to competency in principles of GHE.

Benefits of GHE training for trainees and programs

Studies have shown wide-ranging benefits of GHE educational opportunities in other specialties for both 
trainees and training programs. For trainees, these include enhanced clinical skills, greater appreciation of the importance of public health, positive changes in attitudes and confidence, greater appreciation of cost issues, enhanced cross-cultural sensitivity and communication, diminished reliance on technology, improved understanding of the healthcare challenges in underserved populations and greater exposure to alternative healthcare delivery models. ${ }^{14,30}$ As mentioned, exposure to GHE during training increases the chances a trainee will go on to work with underserved populations and pursue a career in public health or health policy. ${ }^{26}$ For training programs, benefits include increased desirability and recruitment and higher program ranking. ${ }^{31}$

\section{Path forward}

We advocate for the creation of structured GHE opportunities for anesthesia trainees based on three levels of GHE competency. These opportunities should acknowledge the colonial roots of GH and their attendant consequences, ensure mutual benefit through bidirectional partnerships, and educate trainees about local as well as international health disparities. The three levels of GHE competency, modelled on those outlined by the Consortium of Universities for Global Health, ${ }^{29}$ include a basic level for all trainees, an intermediate level for residents wanting to further explore GHE, and an advanced level for fellows or attendings considering dedicating part or all of their careers to GHE (Table 3).

The first level would involve integrating basic GHE education into all North American anesthesia curricula in an effort to increase awareness and baseline knowledge and inspire residents to pursue a career addressing health inequities at home or abroad. The second level, for those with an expressed interest in GHE, would be facilitated by an increase in dedicated resident GHE pathways and formalized opportunities to pursue higher degrees such as
Masters of Public or Global Health during training. The third level would involve the creation of dedicated fellowships for those wanting to dedicate part or all of their careers to GHE. Global health equity fellowships should be as valuable to academic departments as any other subspecialty fellowship. Cornell University and the University of California San Francisco are among the few institutions that have formal GHE programs consistent with the above recommendations, offering didactics for all residents for every year of training, dedicated resident GH tracks with additional training and two to four months of protected scholarly project time, and one- or two-year GH fellowships.

As a first step, we recommend a "basic level" curriculum for all trainees that includes at least one lecture per training year. Though inadequate to achieve competencies, it is feasible for all programs and a starting point for developing more robust learning opportunities. It is also a pragmatic way to identify and support a faculty member to lead a GHE program. Ideally, a broader curriculum would include a combination of lectures, case-based discussions, simulation sessions, and workshops. Topics to be covered should include an introduction to the principles of GHE; the global burden of disease; the global shortfall in surgery and anesthesia provision; pain and analgesia through the lenses of policy, law, global access and abuse; and an introduction to safety net health systems.

Robust frameworks already exist outlining competency domains for non-anesthesia-specific GHE education. ${ }^{29,32}$ These provide a roadmap for any GHE curriculum and need not be superseded. Nevertheless, currently no anesthesia-specific curricula or set of competencies exist at any level of career involvement to complement preexisting GHE syllabi. More work is required to define specific GHE issues related to anesthesia and pain. We invite experts in the field to engage in a structured consensus building process to better define anesthesiaspecific competencies and develop competency assessment

Table 3 Three levels of global health equity competency

\begin{tabular}{lc}
\hline Competency (equivalent CUGH level) \\
\hline $\begin{array}{l}\text { Basic trainee (global citizen } \\
\text { level) }\end{array}$ \\
$\begin{array}{l}\text { Fntermediate trainee (exploratory trainees } \\
\text { level) }\end{array}$ & $\begin{array}{c}\text { For residents wanting to further explore GHE (i.e., through a Master's program or an international or health } \\
\text { disparities project) }\end{array}$ \\
$\begin{array}{l}\text { Advanced trainee (advanced } \\
\text { level) }\end{array}$ & For fellows or attendings considering dedicating part or all of their careers to GHE \\
\end{tabular}

Based on the Consortium of Universities of Global Health (CUGH) proposed levels of Global Health competency ${ }^{29}$

GHE = Global Health Equity . 
tools for the different levels of trainee involvement in GHE.

We acknowledge that introducing any new material into already crowded residency curricula can be challenging. As our specialty continues to develop, demands on programs will increase as the volume of topics to incorporate into curricula expands. This applies to GHE as well as to other subjects, and will require novel teaching strategies to most efficiently deliver existing core material and incorporate new material. Training programs may choose to implement a "flipped classroom" approach that utilizes online modules, videos, and podcasts to supplement in-person case-based or small group learning.

Several institutions have already successfully created online modules for GHE learning. ${ }^{33,34}$ Anesthesia training programs may be able to find such opportunities within their own institution from other disciplines and modify or supplement content to maximally benefit anesthesia trainees without having to reinvent the wheel. The American Board of Pediatrics has created a GHE educational tool, which can be used as general guide for anesthesia educators. ${ }^{35}$ Currently, we are developing open access learning modules with partners that complement existing material. We believe that a formalized and freely available anesthesia-specific curriculum, generated through broader harmonization between academic institutions with defined pathways for different levels of involvement, would streamline the creation of new GHE opportunities for anesthesia trainees. Given the interdependence between anesthesia and surgery, we plan to use this opportunity to increase interdisciplinary collaboration in GHE.

In addition to developing trainee pathways and educational programs, departments should have a mandate to recruit staff with GHE expertise. Departments must show that they value GHE by hiring faculty able to provide training experiences for and mentorship to residents and fellows and develop educational programs. These faculty need to be supported to develop their expertise and to advance the field through scholarly work. Training residents and fellows is necessary but not sufficient.

Finally, we as a specialty must attempt to measure the impact of GHE education initiatives. Currently, many anesthesia training programs track residents' career choices (i.e., which subspecialties they choose and where they practice). The number of trainees who go on to dedicate their careers to underserved populations, whether in an HIC or LMIC, is an equally important metric for program success that we encourage all programs to monitor.

\section{Conclusions}

Anesthesiology is particularly underrepresented in GHE. We are being left behind by other disciplines to the detriment of our patients. Global health equity issues affect everyone everywhere, and are ours collectively to solve. It is time to mobilize and support the next generation of anesthesiologists to address the injustice of health inequity. We need to create providers who engage with their patients' social worlds and take an interest in broader determinants of health. We must give future anesthesiologists the tools to confront GHE challenges wherever they occur and empower them to take a leading role in what will be a defining feature of 21 st century healthcare.

«L'éducation fonctionne soit comme un instrument utilisé pour faciliter l'intégration de la jeune génération dans la logique du système actuel et lui amener la conformité, soit elle devient la pratique de la liberté, moyen par lequel les hommes et les femmes gèrent de manières critique et créative la réalité et découvrent comment participer à la transformation de leur monde. »

- Paulo Freire - Pédagogie des opprimés

L'accès inéquitable à une chirurgie, une anesthésie et à des services de la douleur sécuritaires : voilà l'une des plus importantes crises contemporaines en matière de santé mondiale. Pourtant, la plupart des programmes de formation en anesthésie en Amérique du Nord ne préparent pas adéquatement les futurs anesthésiologistes à reconnaître ou à répondre à cet enjeu de plus en plus criant. Cinq des sept milliards d'habitants de la Terre n'ont pas accès à des services chirurgicaux ou anesthésiques dans un temps et à un coût raisonnables. ${ }^{1}$ Parmi ceux qui ont accès à ces services, plus de 30 millions de personnes sont confrontées chaque année à des coûts de soins de santé catastrophiques (en d'autres termes, lorsque les coûts des soins de santé à payer soi-même mettent les individus et les foyers en difficulté financière). Pour certains, le risque de décéder de complications liées à l'anesthésie peut atteindre 1 sur $100 .^{2}$ L'accès à l'analgésie est tout aussi restreint dans le monde.

Environ $80 \%$ de la population mondiale dispose d'un accès insuffisant ou inexistant à des traitements contre la douleur modérée ou grave, et près de 150 pays ne disposent pas d'un accès fiable à des analgésiques de base tels que la morphine. ${ }^{3}$ Comme l'a souligné l'ancien Président de la Banque mondiale Jim Kim, les iniquités en matière d'accès aux soins chirurgicaux et anesthésiques représentent une grave injustice et mettent en péril le droit fondamental des individus à des soins de santé ainsi que leur capacité de mener « des vies plus en santé et plus productives ». 4 
Les importantes iniquités en matière de soins de santé ne se limitent pas aux pays à revenu faible ou intermédiaire. Dans de nombreux pays à revenu élevé, les populations vulnérables et marginalisées font face à de nombreux obstacles avant d'accéder aux soins de santé, provoquant des disparités alarmantes en matière de santé. ${ }^{5,6}$ Les populations appauvries et rurales ont fréquemment un accès inadéquat aux services chirurgicaux et anesthésiques. ${ }^{7,8}$ Aux États-Unis, la probabilité que les populations autochtones et allophones reçoivent des traitements selon la norme de soins est plus faible. ${ }^{9}$ Dans certaines des plus grandes villes américaines, l'espérance de vie varie de plus de 20 ans entre des quartiers à revenu faible et élevé adjacents. ${ }^{10}$ Une des priorités de tous les programmes de formation devrait être d'encourager les futurs anesthésiologistes à aborder ces problèmes et à y trouver des solutions, tant au niveau local qu'international. $^{11}$

Malgré la popularité croissante de la santé mondiale parmi les résidents en anesthésiologie et les appels urgents à augmenter l'accès aux services d'anesthésie pour la majorité de la population mondiale, il n'existe que peu de programmes de formation en anesthésiologie en Amérique du Nord qui offrent une formation structurée en santé mondiale (c'est-à-dire, des cursus et stages formalisés) afin de préparer les résidents à reconnaître et à gérer ces questions complexes et encourager les carrières en santé mondiale. ${ }^{12}{ }^{15}$ Les résidents intéressés font face à un paysage professionnel disparate et bien souvent déroutant; par conséquent, leur enthousiasme pour la santé mondiale demeure fréquemment sous-développé et sous-utilisé. De nombreux programmes de formation offrent des cours facultatifs dans le cadre desquels les étudiants peuvent faire du travail clinique ou réaliser des projets académiques à court terme dans des pays à revenu faible ou intermédiaire, mais ceux-ci ne sont que rarement accompagnés par une formation en santé mondiale formelle adéquate, ce qui est décevant. ${ }^{13}{ }^{15}$ À l'inverse, d'autres spécialités, telles que la médecine interne et la pédiatrie, disposent de programmes de résidence en santé mondiale bien établis. ${ }^{16,17}$ Près de la moitié de tous les programmes de pédiatrie de l'ACGME (l'organisme responsable de l'accréditation de tous les programmes de formation médicale supérieure aux États-Unis) intègrent la santé mondiale dans leurs programmes de formation. ${ }^{18}$ Récemment, les collèges de chirurgie ont priorisé l'établissement de programmes de formation en santé mondiale et encouragent les carrières dans ce domaine. ${ }^{19}$

Nous demandons l'intégration urgente d'une formation de base en santé mondiale dans tous les programmes de formation en anesthésie en Amérique du Nord, l'expansion des occasions structurées en santé mondiale pour les résidents et fellows souhaitant orienter leur carrière en se concentrant sur les disparités en matière de santé au niveau local ou international, et l'établissement de compétences et de programmes en santé mondiale spécifiques à l'anesthésie. Ces modalités pourront servir d'étapes vers une amélioration de l'accès à des services d'anesthésie, d'analgésie et de chirurgie sécuritaires pour les populations vulnérables et marginalisées dans tous les pays. C'est seulement en offrant une approche plus rigoureuse et structurée de la santé mondiale pendant la formation que l'on peut espérer voir apparaitre des anesthésiologistes conscients de leur environnement et équipés pour devenir des défenseurs efficaces des populations mal desservies qui joueront un rôle plus central dans la création des systèmes de santé futurs.

\section{Qu'entend-on par santé mondiale et par équité en santé mondiale?}

La santé mondiale est un domaine de recherche, d'étude, de revendication, de politiques et de pratiques multidisciplinaire dont l'objectif est d'améliorer la santé mondiale et d'atteindre l'équité en matière de santé à l'échelle mondiale, en se concentrant sur les populations mal desservies. ${ }^{20}$ Malgré de belles intentions, le champ de la santé mondiale et le terme en soi viennent avec leur lot de problèmes et d'interprétations erronées. Auparavant connue sous le nom de "médecine tropicale », la santé mondiale a des racines coloniales qui ont entraîné des défis contemporains, notamment des déséquilibres de pouvoir ayant pour résultat la priorisation des intérêts des pays à revenu élevé ainsi que des bénéfices et une reconnaissance biaisés face aux collaborateurs des pays à revenu élevé.

En outre la santé mondiale est souvent mal comprise en tant que santé internationale, accompagnée de l'idée qu'il faut travailler à l'étranger ou se concentrer sur les défis touchant exclusivement les pays à revenu faible ou intermédiaire. $^{21}$ La santé mondiale transcende les frontières nationales et tente de réduire ou d'éliminer les disparités en matière de santé, peu importe où elles prennent place, sans contrainte géographique. ${ }^{11,21}$

Traditionnellement, la santé mondiale a souvent priorisé certains domaines tels que la santé maternelle et infantile, l'insécurité alimentaire, la pauvreté, les maladies infectieuses et la protection de l'environnement. Jusqu'à récemment la chirurgie, l'anesthésie et la médecine de la douleur n'ont pas été incluses dans les objectifs de la santé mondiale, et ces domaines se retrouvent par conséquent à la traîne derrière la médecine et d'autres disciplines en termes de recherche, de revendication et de formation en santé mondiale.

Récemment, la définition de santé mondiale a été recadrée de façon à mettre l'emphase sur le concept 
d'équité, et a par la suite été renommée par certains «Équité en santé mondiale ». ${ }^{11}$ L'équité est l'absence de différences évitables ou remédiables parmi les groupes d'individus, que ces groupes soient définis d'un point de vue social, économique, démographique ou géographique. Les iniquités en santé sont intrinsèquement injustes et corrigibles. ${ }^{22}$ L'équité en santé mondiale et la pratique de la médecine sociale - un champ de plus en plus important ayant ses bases dans les disparités en matière de santé et dans les déterminants sociaux de santé (Tableau 1) - se recoupent. Selon nous, le terme d'équité en santé mondiale décrit de façon plus précise le domaine, c'est pourquoi nous l'utiliserons pour le reste de ce texte.

En chirurgie et en anesthésie, les initiatives en matière d'équité en santé mondiale se sont surtout manifestées sous forme de «missions ». Cela a entraîné la perception erronée que le rôle principal des anesthésiologistes en équité en santé mondiale s'accomplit par le biais de services cliniques dans un cadre international. Alors que les voyages de mission aident de nombreux patients, leurs détracteurs avancent que certains manquent de transparence, d'imputabilité et de bien-fondé culturel, ${ }^{23}$ et remettent également en question l'éthique et les avantages à long terme de telles missions pour la population locale. ${ }^{24}$ Gardant à l'esprit la définition plus large de l'équité en santé mondiale, et une reconnaissance plus large de l'ampleur des iniquités touchant les soins périopératoires (Tableau 2), nombreux sont ceux qui demandent la mise en place d'approches innovantes et plus durables pour améliorer l'accès mondial à des soins anesthésiques sécuritaires et abordables.

\section{Pourquoi former tous les résidents en équité en santé mondiale?}

L'exposition influence les parcours professionnels

Environ la moitié des résidents en anesthésie continuent leurs études en surspécialité dans des disciplines telles que l'anesthésie cardiaque, pédiatrique, obstétricale et la douleur chronique. ${ }^{25} \mathrm{Il}$ est peu probable qu'ils suivraient de tels parcours sans y avoir été exposés ou avoir reçu de l'enseignement dans ces disciplines pendant leur formation. À l'heure actuelle, aux États-Unis et au Canada, la plupart des résidents en anesthésie ne reçoivent pas d'éducation en équité en santé mondiale pendant leur formation; il est donc peu surprenant qu'ils soient peu nombreux à amorcer des carrières se concentrant sur les populations mal desservies ou sur les disparités en matière de santé, que ce soit au niveau national ou à l'étranger. Une importante proportion de résidents terminent leur formation sans être conscients de l'ampleur des iniquités en santé tant au niveau local que mondial, et ils ne disposent que d'une compréhension limitée des principes d'équité en santé mondiale et des déterminants sociaux de santé. Une exposition à une formation de base en équité en santé mondiale et des stages pendant la formation augmenteraient considérablement la probabilité que des étudiants continuent de consacrer une partie ou l'intégralité de leur carrière à la question des disparités en santé, que ce soit dans les pays à revenu élevé ou dans les pays à revenu faible ou intermédiaire. ${ }^{26}$

Une pertinence dans tous les cadres de pratique

Les iniquités dans l'accès, l'accessibilité économique et la sécurité des soins de santé existent partout et sont pertinentes indépendamment du cadre de pratique ou des

Tableau 1 Définitions : santé mondiale, médecine sociale et déterminants sociaux de la santé

\begin{tabular}{|c|c|}
\hline Discipline & Définition \\
\hline Santé mondiale ${ }^{20}$ & $\begin{array}{l}\text { La santé mondiale est un «domaine d'étude, de recherche et de pratique qui met la priorité sur l'amélioration de la } \\
\text { santé et l'atteinte de l'équité en santé pour tous à l'échelle mondiale. La santé mondiale se concentre sur les } \\
\text { questions de santé, les déterminants et les solutions transnationaux; implique de nombreuses disciplines au sein et } \\
\text { au delà des sciences de la santé et promeut une collaboration interdisciplinaire; et est une synthèse de la } \\
\text { prévention fondée sur la population avec des soins cliniques au niveau individuel. » }\end{array}$ \\
\hline Médecine sociale 22 & $\begin{array}{l}\text { La médecine sociale cherche à comprendre les façons dont les facteurs sociaux influencent la santé, la maladie et les } \\
\text { pratiques médicales. En tant que champ de pratique, elle applique cette compréhension pour aider à la prise de } \\
\text { décisions en matière d'interventions de santé aux niveaux de l'individu, de la communauté et de la société. La } \\
\text { médecine sociale fournit des cadres conceptuels pour aborder les problèmes qui autrement sembleraient insolubles } \\
\text { ou hors du champ de la pratique clinique. }\end{array}$ \\
\hline $\begin{array}{l}\text { Déterminants sociaux de la } \\
\text { santé }\end{array}$ & $\begin{array}{l}\text { Les déterminants sociaux de la santé sont les conditions dans lesquelles les individus sont nés, grandissent, vivent, } \\
\text { travaillent et vieillissent. Ces circonstances sont façonnées par la distribution de l'argent, du pouvoir et des } \\
\text { ressources aux niveaux mondial, national et local. Ces déterminants sociaux de la santé sont principalement } \\
\text { responsables des iniquités en santé - les différences injustes et évitables en statut de santé observées dans et entre } \\
\text { les pays. }\end{array}$ \\
\hline
\end{tabular}


Tableau 2 Dix iniquités importantes en santé mondiale pertinentes à l'anesthésie et à la douleur

1 Cinq milliards d'individus dans le monde n’ont pas accès à des soins chirurgicaux et anesthésiques sécuritaires et abordables

2 Un nombre plus élevé de personnes meurt de conditions traitables par chirurgie et de soins chirurgicaux non sécuritaires que du VIH, de la tuberculose et de la malaria combinés, et pourtant moins de $1 \%$ du fonds de développement pour la santé finance la fourniture de soins anesthésiques et chirurgicaux

3 Seuls $6 \%$ de toutes les opérations sont réalisées dans les pays à revenu faible ou intermédiaire, pourtant la moitié de tous les décès postopératoires surviennent dans ces pays

4 Le taux de mortalité lié à l'anesthésie dans les pays à revenu élevé est d'environ 1:100 000, alors que dans certains pays à revenu faible ou intermédiaire, ce taux peut s'élever à 1:100

5 Plus de 30 millions de personnes sont confrontées à des frais de soins de santé catastrophiques chaque année, particulièrement dans les pays à revenu faible ou intermédiaire

6150 pays n'ont pas d'accès fiable à des analgésiques essentiels tels que la morphine

7 Il existe un écart considérable entre le fardeau mondial de la douleur chronique et les ressources allouées pour s’y attaquer dans les pays à revenu élevé et particulièrement dans les pays à revenu faible ou intermédiaire

8 La probabilité que les populations marginalisées reçoivent des soins anesthésiques « standard », tels que des péridurales obstétricales ou des prescriptions analgésiques, est moins élevée, tant dans les pays à revenu élevé que dans les pays à revenu faible ou intermédiaire

9 Les politiques menées par les pays à revenu élevé en matière de disponibilité mondiale des médicaments anesthésiques et analgésiques limitent de façon disproportionnée l'accès aux médicaments essentiels dans les pays à revenu faible ou intermédiaire

10 La probabilité que les populations rurales et pauvres dans les pays à revenu élevé et à revenu faible ou intermédiaire ait accès à des fournisseurs de soins anesthésiques et en douleur spécialisés est plus faible

$\mathrm{VIH}=$ virus de l'immunodéficience humaine.

projets professionnels futurs (Tableau 2). Afin d'offrir les meilleurs soins possibles à nos patients, nous devons comprendre le rôle puissant que les forces sociales, environnementales, économiques, politiques et culturelles jouent dans la détermination de la santé des patients. Les professionnels de l'anesthésie, comme tous les autres professionnels de la santé, doivent être conscients des facteurs systémiques qui créent et renforcent les iniquités en matière de santé afin de défendre au mieux leurs patients et d'offrir des soins optimaux à toutes les populations de patients.

Exigences à remplir pour respecter les normes actuelles de formation

Aux États-Unis, l'ACGME exige que «tous les étudiants fassent preuve de responsabilité face aux patients, à leurs familles et à la société », et il est attendu qu'ils puissent identifier « les questions importantes pour des populations de patients diverses et la façon dont des ressources limitées peuvent avoir un impact sur les soins aux patients et l'allocation des ressources $» .{ }^{27}$ De la même manière, être un « Promoteur de la santé » figure parmi les compétences de base des anesthésiologistes telles qu'édictées par le Collège royal des médecins et chirurgiens du Canada, ce qui implique que les médecins doivent être capables "d'identifier les déterminants de la santé », de « répondre aux besoins des collectivités ou des populations servies en collaborant avec celles-ci pour promouvoir d'une manière socialement responsable des changements systémiques », de «promouvoir des politiques qui mesurent et soutiennent l'accès aux soins chirurgicaux », et de travailler afin de «soutenir la mobilisation des ressources pour mettre en place des changements $»{ }^{28}$ Selon nous, les exigences de l'ACGME et du Collège royal ne peuvent être respectées que si l'on comprend les principes de base d'équité en santé mondiale grâce à un enseignement formalisé. ${ }^{29}$ Les stages dans les hôpitaux communautaires ou les stages de courte durée dans des environnements où les ressources sont limitées n'équivalent pas à des compétences en matière de principes d'équité en santé mondiale.

Avantages de la formation en équité en santé mondiale pour les résidents et les programmes

Les études ont démontré les avantages majeurs des opportunités de formation en équité en santé mondiale dans d'autres spécialités, tant pour les résidents que pour les programmes de formation. Pour les résidents, ces avantages comprennent des habiletés cliniques améliorées, une meilleure appréciation de l'importance de la santé publique, des changements positifs dans les attitudes et la confiance, une meilleure appréciation des questions de coûts, une sensibilité et une communication interculturelles améliorées, une réduction de la dépendance à la technologie, une meilleure compréhension des défis en matière de soins de santé des populations mal desservies et une plus grande exposition à des modèles alternatifs de fourniture de soins de santé. ${ }^{14,30}$ Comme il a été mentionné, l'exposition à l'équité en santé mondiale pendant la formation augmente les chances qu'un 
résident continue de travailler auprès des populations mal desservies et poursuive son parcours professionnel en santé publique ou en politique sanitaire. ${ }^{26}$ En ce qui a trait aux programmes de formation, les avantages comprennent une plus grande désirabilité et un recrutement plus important, ainsi qu'un positionnement plus élevée du programme. ${ }^{31}$

\section{Voie à suivre}

Nous préconisons la création d'opportunités structurées en équité en santé mondiale pour les résidents en anesthésie qui seront fondées sur trois niveaux de compétences. Ces opportunités devraient tenir compte des racines coloniales de la santé mondiale et des conséquences qui s'ensuivent, garantir des avantages mutuels via des partenariats bidirectionnels, et éduquer les résidents quant aux disparités en matière de santé tant au niveau local qu'international. Les trois niveaux de compétence en équité en santé mondiale, prenant pour modèle ceux décrits par le Consortium des universités pour la santé mondiale (CUGH), ${ }^{29}$ comprennent un niveau de base pour tous, un niveau intermédiaire pour les résidents souhaitant approfondir leurs connaissances, et un niveau avancé pour les fellows ou les médecins en poste envisageant de consacrer une partie ou l'intégralité de leurs carrières à l'équité en santé mondiale (Tableau 3).

Le premier niveau impliquerait l'intégration d'une formation de base en équité en santé mondiale dans tous les programmes d'enseignement d'anesthésie en Amérique du Nord afin de conscientiser les résidents et d'accroître leurs connaissances de base, ainsi que de les inspirer à poursuivre une carrière se concentrant sur les iniquités sanitaires, tant au niveau local qu'international. Le deuxième niveau, destinés à ceux ayant exprimé leur intérêt pour l'équité en santé mondiale, serait facilité par une augmentation des parcours dédiés à l'équité en santé mondiale et par des possibilités formalisées de poursuivre des études supérieures telles qu'une maitrise en santé publique ou mondiale pendant la formation. Le troisième niveau nécessiterait la création de stages spécialisés pour les individus souhaitant consacrer une partie ou l'intégralité de leur carrière à l'équité en santé mondiale. Les fellowships en équité en santé mondiale devraient être aussi prestigieux pour les départements universitaires que les autres fellowships de surspécialité. L'Université Cornell et l'Université de Californie à San Francisco comptent parmi les rares institutions offrant des programmes formels en équité en santé mondiale qui respectent les recommandations susmentionnées, proposant une didactique pour tous les résidents durant chaque année de formation, des parcours en santé mondiale dédiés pour les résidents avec une formation supplémentaire et deux à quatre mois de temps de projet académique protégé, ainsi que des fellowships d'un ou deux ans en santé mondiale.

Comme première étape, nous recommandons un programme de base pour tous les résidents qui comprenne au moins un cours magistral par année de formation. Bien que cela ne suffise pas à développer des compétences, cette approche serait réalisable pour tous les programmes et constituerait un point de départ pour mettre au point des occasions d'apprentissage plus étoffées. Il s'agit également d'une façon pragmatique d'identifier et de soutenir un membre du personnel enseignant afin qu'il prenne la tête d'un programme d'équité en santé mondiale. Dans l'idéal, un programme de cours plus diversifié comprendrait une combinaison de cours magistraux, de discussions fondées sur des cas, de séances de simulation et des ateliers. Les thèmes à aborder devraient comprendre une introduction aux principes de l'équité en santé mondiale; le fardeau mondial de la maladie; les pénuries mondiales dans la fourniture des soins chirurgicaux et anesthésiques; la douleur et l'analgésie selon les perspectives politiques, des lois, de l'accès mondial et des abus; et une introduction aux filets de sécurité des systèmes de santé.

Il existe déjà des cadres robustes décrivant les domaines de compétence à atteindre dans une formation en équité en santé mondiale, mais ils ne sont pas spécifiques à l'anesthésie. ${ }^{29,32}$ Ces cadres constituent une feuille de route pour tout programme en équité en santé mondiale et n'ont pas besoin d'être remplacés. Toutefois, il n'existe à

Tableau 3 Trois niveaux de compétence en équité en santé mondiale

\begin{tabular}{lc}
\hline Compétence (équivalente au niveau du CUGH) \\
\hline $\begin{array}{l}\text { Étudiant de base (niveau du } \\
\text { citoyen mondial) }\end{array}$ & Pour tous les résidents \\
$\begin{array}{l}\text { Étudiant intermédiaire (niveau } \\
\text { exploratoire) }\end{array}$ & $\begin{array}{c}\text { Pour les résidents désirant approfondir l'équité en santé mondiale (par le biais d'une maitrise ou d'un projet } \\
\text { international ou sur les disparités en santé) } \\
\text { Étudiant avancé (niveau avancé) } \\
\begin{array}{c}\text { Pour les fellows ou médecins envisageant de consacrer une partie ou l'intégralité de leur carrière à l'équité } \\
\text { en santé mondiale }\end{array}\end{array}$
\end{tabular}

Basé sur les niveaux proposés de compétence en santé mondiale du Consortium des Universités de santé mondiale (CUGH) ${ }^{29}$ 
l'heure actuelle aucun programme ou ensemble de compétences spécifique à l'anesthésie, à quelque niveau professionnel que ce soit, pour compléter les programmes de cours en équité en santé mondiale préexistants. Il y a encore beaucoup à faire pour définir les questions en équité en santé mondiale spécifiques à l'anesthésie et à la douleur. Nous invitons donc les experts dans ce domaine à prendre part à un processus structuré de création de consensus qui permettra de mieux définir les compétences spécifiques à l'anesthésie et de mettre au point des outils d'évaluation des compétences pour les différents niveaux de formation des étudiants en équité en santé mondiale.

Nous sommes conscients qu'il peut être difficile d'introduire du nouveau matériel éducatif dans des programmes de résidence déjà bien remplis. Alors que notre spécialité continue de se développer, les exigences envers les programmes vont augmenter au fur et à mesure que le volume des thèmes à intégrer dans les programmes universitaires va croissant. Cela s'applique tant à l'équité en santé mondiale qu'à d'autres sujets et nécessitera des stratégies d'enseignement innovatrices afin de livrer de façon plus efficace le matériel de fond existant tout en intégrant le nouveau matériel. Les programmes de formation pourraient décider de mettre en œuvre une approche de «classe inversée » utilisant des modules, vidéos et balados en ligne afin de compléter l'apprentissage fondé sur les cas en personne ou en petit groupe.

Plusieurs institutions ont déjà réussi à créer des modules en ligne pour les apprentissages en équité en santé mondiale. $^{33,34}$ Les programmes de formation en anesthésie pourraient peut-être adapter de telles structures dans leur propre institution à partir d'autres disciplines et modifier ou compléter le contenu afin qu'il soit le plus pertinent pour les résidents en anesthésie sans avoir à réinventer la roue. L'American Board of Pediatrics a créé un outil éducatif qui peut être utilisé comme guide général pour les éducateurs en anesthésie. ${ }^{35}$ À l'heure actuelle, nous mettons au point des modules d'apprentissage en accès libre avec des partenaires qui complèteront le matériel existant. Nous croyons qu'un programme d'enseignement spécifique à l'anesthésie formalisé et en libre accès, créé grâce à une plus grande harmonisation entre les institutions universitaires et proposant des parcours définis selon différents niveaux d'implication, simplifierait la création de nouveaux programmes en équité en santé mondiale pour les résidents en anesthésie. Étant donné l'interdépendance entre l'anesthésie et la chirurgie, nous avons l'intention de nous servir de cette occasion pour accroître la collaboration interdisciplinaire en équité en santé mondiale.

En plus de mettre au point des parcours et des programmes de formation pour les résidents, les départements d'anesthésie devraient également avoir pour mandat de recruter du personnel possédant de l'expérience en équité en santé mondiale. Les départements doivent montrer qu'ils accordent de l'importance à l'équité en santé mondiale en recrutant du personnel capable de fournir des expériences de formation et du mentorat aux résidents et aux fellows tout en développant des programmes de formation. Ces professeurs doivent être soutenus afin qu'ils puissent développer leur expertise et faire avancer le domaine grâce à leurs travaux académiques. Il faut former les résidents et les fellows, mais cela ne suffit pas.

Enfin, en tant que spécialité, nous devons tenter de mesurer l'impact des initiatives de formation en équité en santé mondiale. À l'heure actuelle, de nombreux programmes de formation en anesthésie font le suivi des choix professionnels des résidents (c'est-à-dire, quelles surspécialités ils choisissent et où ils travaillent). Le nombre de résidents qui décident de poursuivre leur carrière en la consacrant aux populations mal desservies, que ce soit dans un pays à revenu élevé, intermédiaire ou faible, constitue une mesure tout aussi importante de la réussite d'un programme, c'est pourquoi nous encourageons tous les programmes à le mesurer.

\section{Conclusion}

L'anesthésiologie est particulièrement sous-représentée en équité en santé mondiale. Nous sommes loin derrière d'autres disciplines, au détriment de nos patients. Les questions d'équité en santé mondiale nous affectent tous, partout dans le monde, et il est de notre responsabilité collective de les résoudre. Il est temps de mobiliser et de soutenir la nouvelle génération d'anesthésiologistes afin de combattre l'injustice de l'iniquité en santé. Nous devons former des fournisseurs de soins de santé qui s'impliquent dans les mondes sociaux de leurs patients et s'intéressent aux déterminants plus globaux de la santé. Nous devons outiller les anesthésiologistes futurs afin qu'ils soient prêts à relever les défis de l'équité en santé mondiale où qu'ils surviennent et leur donner les moyens de prendre la tête de ce qui sera un élément fondamental des soins de santé du $\mathrm{XXI}^{\mathrm{e}}$ siècle.

Acknowledgements Maytinee Lilaonitkul MBBS, University of California San Francisco.

Conflicts of interest None.

Funding statement None.

Editorial responsibility This submission was handled by Dr. Hilary P. Grocott, Editor-in-Chief, Canadian Journal of Anesthesia.

Remerciements Maytinee Lilaonitkul MBBS, Université de Californie à San Francisco. 


\section{Conflit d'intérêt Aucun}

\section{Déclaration de financement Aucune.}

Responsabilité éditoriale Cet article a été traité par Dr Hilary P. Grocott, rédacteur en chef, Journal canadien d'anesthésie.

\section{References}

1. Funk LM, Weiser TG, Berry WR, et al. Global operating theatre distribution and pulse oximetry supply: an estimation from reported data. Lancet 2010; 376: 1055-61.

2. Ouro-Bang'na Maman AF, Agbétra N, Egbohou P, Sama H, Chobli $M$. Perioperative morbidity and mortality in a developing country: experience of Lomé teaching hospital (French). Ann Fr Anesth Reanim 2008; 27: 1030-3.

3. Seya MJ, Gelders SF, Achara OU, Milani B, Scholten WK. A first comparison between the consumption of and the need for opioid analgesics at country, regional, and global levels. J Pain Palliat Care Pharmacother 2011; 25: 6-18.

4. The World Bank. Transcript of recorded video remarks by Jim Yong Kim, President of the World Bank Group, to the Lancet Commission on Global Surgery, January 17, 2014. Available from URL: http://www.globalsurgery.info/wp-content/uploads/ 2014/01/Jim-Kim-Global-Surgery-Transcribed.pdf (accessed March 2020).

5. Hara $K$, Otsubo $T$, Kunisawa S, Imanaka $Y$. Examining sufficiency and equity in the geographic distribution of physicians in Japan: a longitudinal study. BMJ Open 2017; . https://doi.org/10.1136/bmjopen-2016-013922.

6. Nelson AR. Unequal treatment: report of the Institute of Medicine on racial and ethnic disparities in healthcare. Ann Thorac Surg 2017; 76: S1377-81.

7. Daugherty L, Fonseca Benito R, Kumar KB, Michaud PC. Is there a shortage of anesthesia providers in the United States? Santa Monica, CA: RAND Corporation, 2010. Available from URL: https://www.rand.org/pubs/research_briefs/RB9541.html (accessed March 2020).

8. Orser BA, Wilson CR, Rotstein AJ, et al. Improving access to safe anesthetic care in rural and remote communities in affluent countries. Anesth Analg 2019; 129: 294-300.

9. Boffa JD. Cancer care for indigenous Australians. Med J Aust 2008; 188: 560-1.

10. Kopf D, Wolfe D. MAP: What story does your neighborhood's life expectancy tell? Quartz, December 2018. Available from URL: $\quad$ https://qz.com/1462111/map-what-story-does-yourneighborhoods-life-expectancy-tell/ (accessed March 2020).

11. Adams LV, Wagner CM, Nutt CT, Binagwaho A. The future of global health education: training for equity in global health. BMC Med Educ 2016; . https://doi.org/10.1186/s12909-016-0820-0.

12. Evans FM, Mallepally NR, Dubowitz G, Vasilopoulos T, McClain $C D$, Enneking $K$. Factors influencing anesthesia residency selection: impact of global health opportunities. Can J Anesth 2016; 63: 674-81.

13. Kaur G, Tabaie S, Brar J, Tangel V, Pryor KO. Global health education in United States anesthesiology residency programs: a survey of resident opportunities and program director attitudes. BMC Med Educ 2017; . https://doi.org/10.1186/s12909-0171056-3.

14. Tabaie S, Kaur G, Lilaonitkul M, Brumberger E, Dubowitz G, Lipnick MS. Evolving state of global health education for anesthesiology trainees. Curr Anesthesiol Rep 2017; . https:// doi.org/10.1007/s40140-017-0194-9.
15. Global Health Fellowships. Database. Available from URL: http://www.globalhealthfellowships.org/database.html (accessed March 2020).

16. The American Board of Pediatrics. Global Health in Pediatric Education: An Implementation Guide for Program Directors. Available from URL: https://www.abp.org/ghpdguidehome (accessed March 2020).

17. Gladding SP, McGann PT, Summer A, et al. The Collaborative Role of North American Departments of Pediatrics in Global Child Health. Pediatrics 2018; . https://doi.org/10.1542/peds. 2017-2966.

18. Nelson BD, Lee AC, Newby PK, Chamberlin MR, Huang CC. Global health training in pediatric residency programs. Pediatrics 2008; 122: 28-33.

19. Ferrada P, Sakran JV, Vergis A, et al. Above and beyond: A primer for young surgeons interested in global surgery. Bulletin of the American College of Surgeons; February 2017. Available from URL: http://bulletin.facs.org/2017/02/above-and-beyond-aprimer-for-young-surgeons-interested-in-global-surgery/ (accessed March 2020).

20. Dare AJ, Grimes CE, Gillies R, et al. Global surgery: defining an emerging global health field. Lancet 2014; 384: 2245-7.

21. Koplan JP, Bond TC, Merson MH, et al. Towards a common definition of global health. Lancet 2009; 373: 1993-5.

22. Krieger $N$. A glossary for social epidemiology. J Epidemiol Community Health 2001; 55: 693-700.

23. Klar G, Zalan J, Roche AM, Phelan R. Ethical dilemmas in global anesthesia and surgery. Can J Anesth 2018; 65: 861-7.

24. Evans FM, Nabukenya MT. Con: Pure service delivery is no longer needed in global surgical missions. Can J Anesth 2017; 64: 353-7.

25. Warner NS, Warner MA, Eldrige JS, Datta R, Morschler SM, Long TR. Factors Influencing Immediate Post-Residency Career Decisions for Graduating Anesthesiology Residents. J Educ Perioper Med 2018; 20: E616.

26. Thompson MJ, Huntington MK, Hunt DD, Pinsky LE, Brodie JJ. Educational effects of international health electives on U.S. and Canadian medical students and residents: a literature review. Acad Med 2003; 78: 342-7.

27. The Accreditation Council for Graduate Medical Education; The American Board of Anesthesiology. Patient Care-1: Pre-anesthetic Patient Evaluation, Assessment, and Preparation. Available from URL: $\quad$ http://www.acgme.org/Portals/0/PDFs/Milestones/ AnesthesiologyMilestones.pdf (accessed March 2020).

28. Royal College of Physicians and Surgeons of Canada. Anesthesiology Competencies - 2017. Available from URL: http://www.royalcollege.ca/rcsite/documents/ibd/anesthesiologycompetencies-e (accessed March 2020).

29. Jogerst $K$, Callender $B$, Adams $V$, et al. Identifying interprofessional global health competencies for 21st-century health professionals. Ann Glob Health 2015; 81: 239-47.

30. Grudzen CR, Legome E. Loss of international medical experiences: knowledge, attitudes and skills at risk. BMC Med Educ 2007; . https://doi.org/10.1186/1472-6920-7-47.

31. Dey CC, Grabowski JG, Gebreyes K, Hsu E, VanRooyen MJ. Influence of international emergency medicine opportunities on residency program selection. Acad Emerg Med 2002; 9: 679-83.

32. Walpole SC, Shortall C, van Schalkwyk MC, et al. Time to go global: a consultation on global health competencies for postgraduate doctors. Int Health 2016; 8: 317-23.

33. Consortium of Universities for Global Health. Annotated List of Online Global Health Resources. Available from URL: https:// www.cugh.org/content/annotated-list-online-global-healthresources (accessed March 2020).

34. Kaur G. Essentials of Global Health and Human Rights for Physicians. In: Apple iBook Store - April 2019. Available from 
URL: https://books.apple.com/us/book/essentials-global-healthhuman-rights-for-physicians/id1459571669 (accessed March 2020).

35. The American Board of Pediatrics. Global health in pediatric education: an implementation guide for program directors - 2018. Available from URL: https://www.abp.org/sites/abp/files/pdf/ globalhealthinpediatriceducationimplementationguideforprogram directors.pdf (accessed March 2020).

Publisher's Note Springer Nature remains neutral with regard to jurisdictional claims in published maps and institutional affiliations. 